\title{
Oral Communication and Scientific Nomenclature on Medicinal Wild Plants in Culiacan, Sinaloa, México: An Educational Proposal
}

\author{
Hipólito Aguiar Hernández ${ }^{1}$, Leopoldo Partida Ruvalcaba ${ }^{2 *}$, Jacobo Enrique Cruz Ortega1 \\ ${ }^{1}$ Facultad de Agronomía, Universidad Autónoma de Sinaloa, Culiacán de Rosales, México \\ ${ }^{2}$ Universidad Tecnológica de Culiacán, Culiacán de Rosales, México \\ Email: *parpolo@yahoo.com.mx
}

How to cite this paper: Hernández, H.A., Ruvalcaba, L.P. and Ortega, J.E.C. (2016) Oral Communication and Scientific Nomenclature on Medicinal Wild Plants in Culiacan, Sinaloa, México: An Educational Proposal. Open Access Library Journal, 3: e3075. http://dx.doi.org/10.4236/oalib.1103075

Received: September 19, 2016

Accepted: October 10, 2016

Published: October 13, 2016

Copyright $\odot 2016$ by authors and Open Access Library Inc.

This work is licensed under the Creative Commons Attribution International

License (CC BY 4.0).

http://creativecommons.org/licenses/by/4.0/

\section{cc) (i) Open Access}

\begin{abstract}
This research was done with objetive to know if oral communication among members of communities in the municipality of Culiacan, Sinaloa, Mexico, is the principal means of transmitting knowledge regarding medicinal wild plants often used by people of the same communities, and identify said plants by the name scientific to the benefit of the citizens in general. The resources or techniques used to compile the information required were field observation and interview. The information was obtained by applying interviews for asking about the species known as medicinal plants and used in the community. They were carried out field trips to different communities in the municipality of Culiacan, looking for information of species used for medicinal purposes. The samples were collected when necessary and materials for photographic collections of plants were studied, as well as respondents. In each community five people were interviewed, including the old, men or women, who were selected randomly, and young people who have knowledge about the use of plants for medicinal purposes. The collection of plants was made without adversely affecting the conservation of the species studied and used as medicinal. The identification of the collections was made by consulting literature. Oral expression is the predominant form of transmitting knowledge about medicinal plants, wild or cultivated, each with scientific nomenclature, and the inhabitants of the communities known, of manner own or through others, what wild plants have properties to cure diseases.
\end{abstract}

\section{Subject Areas}

Plant Science

Keywords

Form of Communication, Medicinal Plants, Common Name, Scientific Name 


\section{Introduction}

El uso de plantas medicinales es resultado de la experiencia e íntimo contacto con la naturaleza que el hombre ha acumulado por generaciones, así como de la convivencia entre las culturas de diferentes pueblos. Este saber ha permitido que sobrevivan comunidades que habitan en lugares apartados, donde hay carencias de servicios médicos y donde las parteras, "yerberos" y curanderos son los principales responsables de la salud de los habitantes [1].

Actualmente existe un interés creciente por la medicina alternativa para curar numerosos padecimientos y enfermedades que afectan al hombre, por lo que investigaciones que tengan como objetivo el cultivo, estudio y procesamiento de plantas medicinales con fines terapéuticos se consideran estratégicas e importantes [2]. En las áreas marginadas existen grupos indígenas con grandes conocimientos de la herbolaria medicinal y del ambiente donde se desarrollan estas plantas. Tales conocimientos acumulados por muchas generaciones constituyen la base de la biodiversidad cultural global y del uso sustentable de estos recursos [3] [4]. Para estudiar las plantas medicinales es indispensable la exploración etnobotánica, que incluye la colección, propagación y conservación de las especies [5] [6].

De acuerdo con García et al. [7], en artículos científicos de la época colonial y neocolonial de Cuba, se encontró información acerca de 38 especies de plantas medicinales para tratar problemas de salud, de las cuales: Allium sativum L. (ajo), Allium cepa L. (cebolla), Carica papaya L. (fruta bomba), Coffea arabica L. (café), Cucurbita pepo L. (calabaza) y Mangifera indica L. (mango), también se emplean en el Sistema Nacional de Salud de Cuba [8] y fueron ratificadas en el Programa Nacional de Medicina Tradicional y Natural del mismo país [9]. Todas éllas y, además, Jatropha curcas L. (piñón de botija) se encuentran entre las especies recomendadas por el Programa de Investigaciones y Difusión de Usos de Plantas Medicinales del Caribe [10]. Asimismo, se encontró que a pesar de los esfuerzos de los científicos cubanos de dicha época, con respecto a las investigaciones de las plantas medicinales para demostrar sus virtudes terapéuticas con vistas a su aplicación en la medicina y la farmacia, y aportar elementos esenciales para contribuir a la conformación de la flora médica indígena del país, en la literatura revisada de esta época se publicaba poca información sobre el uso de las plantas medicinales y sus estudios [7].

En México, alrededor de 4000 especies de plantas con flores (aproximadamente 15\% de la flora total) tienen atributos medicinales, es decir que más o menos una de cada siete especies posee alguna propiedad curativa. Sin embargo, se estima que la validación química, farmacológica y biomédica de los principios activos que contienen se ha llevado a cabo sólo en $5 \%$ de estas especies. Los antiguos pobladores de nuestro territorio desarrollaron una de las herbolarias más complejas del mundo, debido a la riqueza cultural y étnica que alcanzaron, por lo que desde tiempos prehispánicos diferentes grupos étnicos han usado plantas con fines medicinales [11].

México es uno de los países de América con mayor tradición ancestral y riqueza en el uso de la herbolaria medicinal; sin embargo, son pocas las investigaciones en el uso y 
manejo de las plantas medicinales y, en consecuencia, es escasa la información etnobotánica sobre este tema [5] [12]-[14]. La información que se pueda recopilar en las diversas regiones del país tendría relevancia etnobotánica y serviría para definir estrategias que mejoren el aprovechamiento y manejo de los recursos de la flora medicinal [1]. En varios municipios de Tabasco se ha iniciado un proceso de recuperación del germoplasma medicinal y de sistematización del conocimiento mediante la publicación de manuales y artículos científicos donde se documenta la experiencia acumulada en el uso de la medicina naturista [15].

En la Ranchería Corregidora Ortiz $1^{\text {ra }}$ Sección se encontraron 112 especies que se utilizan para tratar 42 malestares. Estas especies se agrupan en 57 familias botánicas, de las cuales las más representativas, con tres a diez especies por familia, son: Asteraceae (10), Lamiaceae (7), Fabaceae (5), Malvaceae (5), Rutaceae (5), Bignoniaceae (4), Euphorbiaceae (4), Verbenaceae (4), Amarillidaceae (3), Piperaceae (3) y Solanaceae (3). Por tipo biológico $42 \%$ son herbáceas, $24 \%$ arbustivas, $25 \%$ arbóreas, $7 \%$ trepadoras y $2 \%$ de tubérculos [1]. El conocimiento ancestral proviene de los abuelos, en $36 \%$, seguido de los padres con $27 \%$, de los hijos y vecinos $28 \%$, y el resto de otras fuentes. La mayoría de las plantas crecen en la propia comunidad, y muchas de ellas se utilizan para elaborar biopesticidas naturales [1].

A través de una revisión bibliográfica, Fresquet [16] reportó que en Valencia, España, son 146 las especies de plantas que se usan con fines medicinales, entre otros usos. Adicionalmente, encontró que 13 son las más utilizadas, seguidas de ocho especies en un segundo nivel de utilización y posteriormente otro grupo de 21 especies. Todas ellas fueron mencionadas tanto en Rivera Baja, como en el Parque Natural de Ia Albufera y Rivera Alta, de tal manera que en Valencia fueron 104 las menos mencionadas por parte de las personas entrevistadas, 126 en Rivera Baja y 110 en el Parque Natural de la Albufera. Este trabajo de revisión también permitió detectar que 66 especies encontradas en Valencia no figuran en las otras tres zonas, 36 referidas en Valencia y el Parque Natural de la Albufera no figuraron en Ribera Alta y 19 mencionadas en Valencia y Rivera Alta fueron omitidas en el Parque Natural de la Albufera.

La tradición oral ha sido el mejor método para alimentar la memoria del pasado entre los pueblos ágrafos, y casi fue la única forma de comunicación empleada por el hombre prehistórico y, por supuesto, entre los pobladores del Neolítico y Arqueolítico mesoamericano, entre quienes el conocimiento se trasmitía de padres a hijos, de matriarcas y patriarcas a la descendencia, en general eran las personas ancianas y sabias las que trasmitían el conocimiento del pasado, como el de las plantas medicinales, a las nuevas generaciones [17].

Los nombres científicos, a diferencia de los comunes, son universales y no están restringidos a una región en su lengua local. Muchas plantas, generalmente de amplia distribución o de uso extendido y por tanto muy conocidas, pueden tener más de una docena de nombres comunes. Por otra parte, dos o más especies de plantas, incluso no emparentadas o totalmente diferentes, pueden tener el mismo nombre común; en contraste, de muchas especies, en particular las que no tienen un uso directo o las que 
son raras o poco conocidas [11].

De acuerdo a los registros existentes, las hierbas medicinales tienen una larga historia que se pierde a menudo entre el mito y la leyenda, sin embargo, las actuales investigaciones han puesto en evidencia que un gran número de plantas utilizadas por nuestros antepasados, o que forman parte de la medicina tradicional de muchos pueblos actuales, son capaces efectivamente de aportar beneficios a nuestra salud [18].

Los aztecas desarrollaron una verdadera práctica médica basada en el conocimiento de las propiedades curativas de las plantas, en la actualidad la medicina moderna alopática sigue los pasos a la estadounidense, el comercialismo estadounidense ha invadido México, sin embargo, es alentador saber que numerosos países estudian las plantas con la finalidad de encontrar en éllas los remedios para las enfermedades [19].

Por otra parte, recientemente se logró la caracterización fitoquímica y la determinación de cómo el cromo de hojas de tronadora [ Tecoma stans L. (Juss. ex Kunth)], ocasionó efecto hipoglucemiante en animales con diabetes mellitus inducida [20].

Un grupo de investigadores de la Facultad de Química y Ciencias Biológicas de la Universidad Autónoma de Sinaloa ha emprendido el estudio sobre plantas nativas y su probable utilidad en la salud humana. De este esfuerzo han surgido algunas publicaciones, entre ellas la de Camacho et al. [21], quienes encuentran propiedades antifungales en el arrayán [Psidium sartorianum (O. Berg) Nied.] y la de Pío-León et al. [22] que encontraron cualidades nutricionales y antioxidantes en semillas de frutilla (Ebenopsis caesalpinioides Standl.).

También se llevó a cabo una investigación que muestra las propiedades fisicoquímicas y nutricionales de la fruta uvalama (Vitex mollis Kunth). Dicho estudio refiere que la parte comestible se debe incluir en la dieta mexicana, como una fuente importante de nutrientes y compuestos beneficiosos para la salud; en consecuencia, la conservación de esta especie podría dar lugar a nuevas investigaciones y otros beneficios prácticos [23].

Aún reconociendo que actualmente existen aportaciones importantes, como las anteriormente mencionadas, desde la investigación etnobotánica y las instituciones educativas relacionadas con el área agropecuaria para recuperar, proteger y difundir los conocimientos de la herbolaria y de la medicina tradicional, en lo general dichas aportaciones son escasas. Particularmente en el estado de Sinaloa son pocos los estudios al respecto, destacando una investigación importante por su rigurosidad y contenido científico, realizada por el Dr. Rito Vega Aviña en su obra titulada Flora en Sinaloa [24].

El objetivo de esta investigación fue determinar si la comunicación oral entre los miembros de comunidades es el principal medio para transmitir conocimiento acerca de las plantas silvestres medicinales que suelen usar los pobladores de las mismas, e identificar por su nombre científico a dichas plantas para beneficio de los ciudadanos en general.

\section{Materiales Y Métodos}

La presente investigación tiene un enfoque cualitativo descriptivo, en cuanto que recoge 
en su propio contexto sociocultural e histórico, la opinión de los pobladores de diferentes comunidades del municipio de Culiacán, Sinaloa, México, que conocen las propiedades de las plantas silvestres medicinales y las emplean tradicionalmente para curar sus enfermedades. Los recursos o técnicas utilizadas para compilar la información requerida fueron la observación de campo y la entrevista.

Para éllo se incluyó información ecológica y etnoecológica de las especies y los ambientes, para identificar cómo los recursos florísticos medicinales son percibidos, conservados y aprovechados. Se trabajó dentro del esquema de la metodología participativa, al considerar que los sujetos poseedores del conocimiento forman parte integral del estudio, y aportan toda su información en un clima de respeto mutuo y revaloración de su conocimiento.

Con la investigación de campo se obtuvo información y opiniones directamente por parte de los sujetos que formaban parte del ámbito bajo investigación y en el propio contexto donde ocurrieron los hechos. De tal manera que se hicieron encuestas en 18 comunidades, donde se entrevistó a 57 hombres y 37 mujeres con edades entre 24 y 84 años, que incluyó amas de casa, agricultores, jornaleros, ganaderos, pescadores y vaqueros.

El municipio de Culiacán se localiza en la parte central del Estado de Sinaloa, México, entre las coordenadas $24^{\circ} 48^{\prime} 15^{\prime \prime} \mathrm{N}$ y $107^{\circ} 25^{\prime} 52^{\prime \prime} \mathrm{O}$. Su territorio se extiende en $4758.9 \mathrm{~km}^{2}$, es decir, aglutina dentro de sus límites al $8.2 \%$ de la superficie del estado, lo que lo convierte en el tercer municipio más extenso, esto es después de los municipios de Sinaloa de Leyva y Badiraguato.

Las características geológicas del municipio de Culiacán son: franja costera que está formada por capas recientes del pleistoceno y formaciones geológicas del principio de la era cuaternaria. La región central por la naturaleza rocosa del cenozoico y las partes elevadas de la sierra, están compuestas principalmente por rocas metamórficas de la era mesozoica. Predominan los suelos feozem, vertisol, regosol y cambisol, la mayor parte del suelo es de uso agrícola [25].

El municipio tiene temperatura media anual de $25^{\circ} \mathrm{C}$, con precipitación pluvial promedio de 658 milímetros al año, el clima es húmedo y caliente en verano $\left(36^{\circ} \mathrm{C}\right)$, mientras que en invierno la temperatura es agradable $\left(10.5^{\circ} \mathrm{C}\right)$, con escasas precipitaciones, los vientos dominantes se desplazan en dirección noroeste, con velocidad aproximada de dos metros por segundo [25].

En el municipio es abundante la selva baja caducifolia, existiendo también bosque de encino y de pino-encino. En el área costera se encuentra vegetación halófila y manglar. De conformidad al inventario nacional forestal del año 2000, el estado de Sinaloa cuenta con 5,832,800 ha de superficie territorial [25].

Para esta investigación se identificó la zona de estudio y se establecieron los contactos necesarios con informantes clave por comunidad, a fin de construir la estrategia que permitió realizar este trabajo con enfoque cualitativo, que consistió en la intención de observar los fenómenos que interesaban, tal y como se dieron en su contexto natural, para luego analizarlos. Esto implicó el contacto directo con la realidad, el empleo de la 
observación, entrevistas y encuestas que permitieron describir sus experiencias en sus procesos de planificación en los que hayan intervenido o formado parte, así como las consideraciones sobre los resultados obtenidos.

El aspecto documental de la presente investigación consistió en hacer una revisión de lo que se ha investigado en el municipio sobre plantas silvestres medicinales en revistas y literatura especializada, para conocer la situación que guarda el conocimiento de este tipo de plantas, desde la conservación de la biodiversidad.

La metodología cualitativa permitió la perspectiva teórica centrada en la Etnobotánica y la Pedagogía Humanista, la cual consistió en obtener de la población la mayor información, a partir de su participación activa en la etapa de recopilación de datos. La información se obtuvo mediante la aplicación de entrevistas para preguntar sobre las especies conocidas como plantas medicinales y usadas en la comunidad. Se llevaron a cabo salidas de campo a las diferentes comunidades del municipio de Culiacán, para la búsqueda de información de especies utilizadas con fines medicinales. Se colectaron muestras cuando fue necesario y se hizo acopio de material fotográfico de plantas estudiadas, así como de personas encuestadas. En cada comunidad se realizaron entrevistas a cinco personas mayores hombres o mujeres seleccionadas de manera aleatoria, así como a personas jóvenes que tuvieran conocimientos respecto al uso de plantas con fines medicinales. Se hicieron colectas de las plantas sin atentar contra la conservación de las especies estudiadas y más utilizadas como medicinales. La identificación de las colectas se hizo mediante la consulta de literatura especializada.

\section{Resultados Y Discusión}

En la Table 1 se indica la relación de plantas medicinales que se formó al hacer las entrevistas y encuestas en las comunidades del Municipio de Culiacán, que fueron objeto de estudio en esta investigación.

A través de la pregunta ¿Conoce usted plantas medicinales que se encuentren en el campo? hecha a 90 sujetos informantes, se pudo notar que de manera general las personas sí tienen conocimiento que existen plantas medicinales, y ante la pregunta ¿cuáles son? se elaboró el cuadro en cuestión.

Ante las preguntas ¿qué tipo de enfermedades suelen padecer y con qué plantas las han solucionado? y ¿ cómo conoció usted las propiedades curativas de la plantas medicinales que existen en su comunidad?, de 90 encuestados, el 60.3\% (61 entrevistados) respondieron si conocer el uso curativo de las plantas de acuerdo a diferentes enfermedades que los afectan, mientras que el 39.7\% (29 entrevistados) dijeron desconocer su uso; no obstante, dijeron si tener nociones de algunas plantas medicinales, cuyos conocimientos fueron adquiridos de manera oral de sus ancestros, y que los han recibido por generaciones a través de la experiencia que los pobladores de estos lugares han adquirido ante situaciones de problemas de salud, mismos que han sido tratados con este tipo de plantas y que en consecuencia las han clasificado como medicinales por los resultados favorables de salud que han obtenido. Son en este caso, conocimientos empíricos que no están muchas de las veces documentados, pero que se han adquirido 
Table 1. Plantas medicinales más comunes que mencionaron los pobladores en las diferentes comunidades del municipio de Culiacán, Sinaloa, México.

\begin{tabular}{|c|c|c|}
\hline 1. Aguama & 25. Guaco & 49. Palo blanco \\
\hline 2. Apoma & 26. Gualámo & 50. Palo mulato \\
\hline 3. Apomo & 27. Gatuño & 51. Papachio \\
\hline 4. Arrayán & 28. Golondrina & 52. Pimientilla \\
\hline 5. Ayale & 29. Guamúchil & 53. Poleo \\
\hline 6. Batoco & 30. Guasima & 54. Palo dulce \\
\hline 7. Brasil & 31. Guayabo & 55. Romero \\
\hline 8. Bugambilia & 32. Buirote cachora & 56. Rosamarilla \\
\hline 9. Cacarahua & 33. Hierba de la víbora & 57. Sacamanteca \\
\hline 10. Capule & 34. Hierba de manzo & 58. Sangregado \\
\hline 11. Cardon & 35. Hierba de soldado & 59. Solda con salda \\
\hline 12. Chapote & 36. Hierba del golpe & 60. Sinvergüenza \\
\hline 13. Chicayota & 37. Hierba del indio & 61. Tatachinole \\
\hline 14. Chicura & 38. Higuerilla & 62. Taray \\
\hline 15. Choya & 39. Huichapore & 63. Tepehuaje \\
\hline 16. Ciática & 40. Huizache & 64. Toloache \\
\hline 17. Ciruelo coyote & 41. Limón & 65. Tripa de zopilote \\
\hline 18. Cola de caballo & 42. Maguey & 66. Uvalámo \\
\hline 19. Confitón & 43. Mangle & 67. Vara blanca \\
\hline 20. Copalquín & 44. Mauto & 68. Vinorama \\
\hline 21. Coronita & 45. Mezquite & 69. Zapote \\
\hline 22. Cuajilote & 46. Micle & 70. Zorrillo \\
\hline 23. Damiana & 47. Negrito & \\
\hline 24. Estafiate & 48. Nopal & \\
\hline
\end{tabular}

por la tradición y la trasmisión oral.

$\mathrm{Al}$ respecto, existe mucha literatura donde se informa sobre este tipo de procesos y formas de cultura tradicional, que se dan en las poblaciones indígenas y rurales en muchas regiones del mundo, donde no existen condiciones para atender de otra manera sus enfermedades, sino a través de conocimientos herbolarios que han adquirido, y que hoy en día son recuperados como parte de la medicina alternativa e integrativa que alivia los males.

$\mathrm{Al}$ hacer la pregunta ¿a dónde recurre para obtener las plantas medicinales y curarse en casa? Las respuestas fueron que las adquieren en el campo, en la casa, en el monte, en las lomas, en el patio de la casa, en los cerros, barrancos húmedos, las encarga a otras personas.

De manera general se observa que esta forma de curar sus enfermedades, que existe 
en la población rural, en las comunidades situadas en las zonas de los altos en el municipio de Culiacán, las cuales corresponden a las sectores más pobres y desprotegidos de la población, son parte de la cultura y tradiciones de las comunidades que se desarrollan sin mediar cierto tipo de relaciones comerciales como resultado de sus propias condiciones de existencia. Se puede constatar que la mayoría de medicamentos que son utilizados en estos lugares son adquiridos de manera directa y natural en los montes, en los barrancos y en los arroyos de sus lugares de origen.

Cuando se planteó la pregunta ¿su familia siempre cuenta con plantas medicinales en casa, de qué forma, secas o frescas y cuáles son? Las respuestas de 66 personas (73.3\%) de las 90 entrevistadas, respondieron afirmativamente, 16 (17.8\%) que no siempre cuentan con éllas, en tanto que ocho personas (8.9\%) no respondieron al cuestionamiento. De acuerdo a las respuestas obtenidas se puede inferir que en estas regiones de Sinaloa, no obstante la cercanía a la capital, un alto porcentaje de la población, $80 \%$ en promedio, recurren a medicamentos naturales derivados de plantas medicinales que existen en sus lugares de origen para atenderse cierto tipo de enfermedades y cuentan con estos remedios en sus propias casas. En cuanto a si las plantas medicinales que tienen en sus casas son frescas o secas, el $60 \%$ dijeron que son frescas, $15 \%$ secas y $15 \%$ dijo que en ambas formas. Entre las plantas más comunes utilizadas por los pobladores para aliviar algunas enfermedades, se encuentran 44 especies, entre las cuales sobresalen el cardón, brasil, tachinole, entre otras.

Cuando se les preguntó ¿de las plantas que ya mencionó cuáles compra y cuáles cultiva? El 95\% de las respuestas fue que las compran, el 2\% que si las compran, el 2\% respondieron que algunas veces las compran, mientras que el $1 \%$ dijo que no las compra, sino más bien las vende. Según las respuestas de los pobladores, las plantas medicinales que éllos introducen de otras partes del país y/o cultivan, son las que se indican en la Table 2.

La mayor parte de los pobladores de estas comunidades conocen las plantas medicinales que existen de manera silvestre en estas zonas del estado, así como el uso que se les da y para qué tipo de enfermedades se emplean, este dato está documentado en uno de los cuadros anteriores. Se observa, que en este estudio se registra que el conocimiento de este tipo de saberes medicinales no son únicamente en las mujeres o en los ancianos, en nuestra investigación encontramos que los hombres del campo en vida útil tienen amplios conocimientos empíricos al respecto, lo cual quizás se deba a

Table 2. Plantas medicinales introducidas y nativas que son cultivadas por los pobladores del Municipio de Culiacán, Sinaloa, México.

\begin{tabular}{ccccc}
\hline \multicolumn{2}{c}{ Plantas medicinales introducidas } & \multicolumn{2}{c}{ Plantas medicinales nativas } \\
\hline Albahaca & Maíz & Sábila & Cardón & Nopal \\
\hline Bugambilia & Manzanilla & Tomate & Confitón & Palo mulato \\
Laurel & Poleo & Valeriana & Copalquin & Toloache \\
Lechuga & Romero & & Estafiate & \\
Linaza & Ruda & & Micle & \\
\hline
\end{tabular}


que son éllos quienes exploran directamente sus tierras y conocen las diferentes especies vegetales que ahí se desarrollan.

Se observa que los conocimientos de herbolaria que tienen los habitantes de las comunidades en cuestión, son conocimientos empíricos que no van más allá de los adquiridos por la experiencia y sus prácticas cotidianas. Cuando se trata de explicar las razones por las cuales las plantas medicinales ayudan para aliviar ciertas enfermedades, las respuestas son demasiado simples y superficiales, esto obedece al nivel de formación cultural y académica que poseen, que no les permite construir una respuesta más teórica y científica. Sin embargo dichos conocimientos no dejan de ser muy valiosos y se deben conservar y estudiar en una perspectiva científica por los académicos y las instituciones educativas relacionadas con estas áreas del conocimiento, con el propósito de que estos saberes no se pierdan.

El nombre común y científico de las plantas medicinales (Table 3) que usan los pobladores del municipio de Culiacán, Sinaloa, es importante para los investigadores y los académicos de los niveles educativos de primaria, secundaria, preparatoria, profesional y postgrado.

De tal manera que los nombres comunes y científicos que se proporcionan en el cuadro anterior, pueden servir para otras investigaciones por académicos e investigadores, o para que los usuarios los puedan encontrar con más facilidad en el mercado y puedan tener acceso más rápido a éllos para tratar la o las enfermedades que les aquejen.

Estos resultados coinciden con los de Rojas [17], toda vez que este autor ha reportado que la tradición oral ha sido el mejor método de comunicación empleada por el hombre prehistórico y, por supuesto, entre los pobladores del Neolítico y Arqueolítico mesoamericano, quienes de padres a hijos, de matriarcas y patriarcas trasmitían sus conocimientos y experiencias a las nuevas generaciones. Asimismo, con los de Gómez [1], quien ha reportado que el conocimiento ancestral proviene de los abuelos, padres, hijos, vecinos y otras fuentes, y que la mayoría de las plantas crecen en la propia comunidad, y que el uso de las plantas medicinales es resultado de la experiencia que el hombre ha acumulado por generaciones. Además, corroboran las aportaciones de las investigaciones que han dado evidencias de que las plantas utilizadas por los antepasados son efectivas para ocasionar beneficios a la salud del hombre, como lo reportó Ramírez [18].

También confirman que en las zonas marginadas existen grupos de personas con conocimientos de la herbolaria medicinal y del ambiente donde se desarrollan estas plantas. Conocimientos acumulados por muchas generaciones que constituyen la base de la biodiversidad cultural, como lo reportaron Leonti et al. [3] y Canales et al. [4]. Para lo cual, según Hernández et al. [5] y Ocampo [6], es indispensable la exploración etnobotánica, que incluye la colección, propagación y conservación de las especies.

También concuerdan con los de García [19], ya que éste refiere que los aztecas desarrollaron la práctica médica con base en el conocimiento de las propiedades curativas de las plantas, y que en muchos países se estudian las plantas con la finalidad de encontrar en éllas los remedios para las enfermedades. 
Table 3. Nombre común y científico de las plantas medicinales que usan los pobladores de Culiacán, Sinaloa, México.

\begin{tabular}{|c|c|c|}
\hline No. & Nombre común & Nombre científico \\
\hline 1 & Aguama & Bromelia pinguin \\
\hline 2 & Antena & Lantana cámara \\
\hline 3 & Apomo & Brosimum alicastrum \\
\hline 4 & Arrayán & Psidium sartorianum \\
\hline 5 & Ayale, Tecomate & Crescentia alata \\
\hline 6 & Bejuco tres caras & Serjania sp. \\
\hline 7 & Berraco, Tapaco & Tabernaemontana amygdalifolia \\
\hline 8 & Brasil & Haematoxylon brasiletto \\
\hline 9 & Brea & Cercidium praecox \\
\hline 10 & Buena mujer & Solanum \\
\hline 11 & Cacachila & Colubrina glomerata \\
\hline 12 & Cacarahua & Vallesia glabra \\
\hline 13 & Camote amargo & Dioscorea \\
\hline 14 & Candelilla & Pedilanthus macrocarpus \\
\hline 15 & Capule & Ficus sp. \\
\hline 16 & Cardón & Pachycereus pecten-aboriginum \\
\hline 17 & Carrizo & Arundo donax \\
\hline 18 & Ciruelo coyote & Spondias \\
\hline 19 & Cola de caballo & Equisetum $s p$ \\
\hline 20 & Compio & Combretum farinosum \\
\hline 21 & Confiton & Tournefortia hartwegiana \\
\hline 22 & Copal, Jiote & Bursera simaruba \\
\hline 23 & Copalquín & Coutarea pterosperma \\
\hline 24 & Coronita & Antigonum leptopus \\
\hline 25 & Coscomate & Physalis \\
\hline 26 & Cuajilote & Parmentiera aculeata \\
\hline 27 & Chapote & Diospyros \\
\hline 28 & Chicayota & Cucurbita angyrosperma \\
\hline 29 & Chicura & Franseria ambrosioides \\
\hline 30 & Choya & Cylindropuntia sp. \\
\hline 31 & Damiana & Turnera ulmifolia \\
\hline 32 & Doradilla, Flor de piedra & Sellaginella pallescens. \\
\hline 33 & Ébano & Caesalpinia sclerocarpa \\
\hline 34 & Estafiate & Ambrosia artemisifolia \\
\hline 35 & Gallinita & Mascagnia macroptera \\
\hline
\end{tabular}




\section{Continued}

\begin{tabular}{|c|c|c|}
\hline 36 & Gatuño & Mimosa biuncifera \\
\hline 37 & Golondrina & Euphorbia hirta \\
\hline 38 & Gordolobo & Gnaphalium spp \\
\hline 39 & Grama, Pata de gallo & Cynodon dactylon \\
\hline 40 & Guaco & Aristolochia taliscana \\
\hline 41 & Guachapore & Cenchrus echinatus \\
\hline 42 & Guamúchil & Pithecellobium dulce \\
\hline 43 & Guareque & Maximowisda sonorae \\
\hline 44 & Guásima & Guazuma ulmifolia \\
\hline 45 & Guayacán & Guaiacum palmeri \\
\hline 46 & Guirote de cachora & Cardiospermum halicacabum \\
\hline 47 & Hierba de la víbora & Zornia sp. \\
\hline 48 & Hierba del golpe & Oenothera $s p$ \\
\hline 49 & Hierba del indio & Aristolochia quercetorum \\
\hline 50 & Hierba del manso & Anemopsis califórnica \\
\hline 51 & Hierba del soldado & Arenaria sp. \\
\hline 52 & Hierba santa & Piper auritum \\
\hline 53 & Higuerilla & Ricinus communis \\
\hline 54 & Huanacaxtle & Enterolobium ciclocarpum \\
\hline 55 & Huizache & Acacia cymbispina \\
\hline 56 & Maguey & Agave sp. \\
\hline 57 & Mangle & Rizophora mangle \\
\hline 58 & Mastranzo & Mentha suaveolens \\
\hline 59 & Matanene & Mascagnia macroptera \\
\hline 60 & Mauto & Lysiloma divaricada \\
\hline 61 & Mezquite & Prosopis juliflora \\
\hline 62 & Micle & Jacobinia spicigera \\
\hline 63 & Muerdago, Tofel & Psittacanthus calyculathus \\
\hline 64 & Nanche, Nanchi & Byrsonima crassifolia \\
\hline 65 & Negrito & Karwuinskia humboldtiana \\
\hline 66 & Ocote & Pinus oocarpa \\
\hline 67 & Ojo de zanate & Rhynchosia \\
\hline 68 & Otate & Bambusa vulgaris \\
\hline 69 & Palo blanco & Ipomoea arborescens \\
\hline 70 & Palo colorado & Caesalpinia platyloba \\
\hline 71 & Palo dulce & Eysenhartia polystachya \\
\hline 72 & Palo mulato & Bursera \\
\hline
\end{tabular}




\section{Continued}

\begin{tabular}{|c|c|c|}
\hline 73 & Papache & Randia echinocarpa \\
\hline 74 & Papachío & Randia mitis \\
\hline 75 & Pata de león, Mano de león & Philodendron sp. \\
\hline 76 & Pimientilla & Parthenium hysterophorus \\
\hline 77 & Pino & Pinus sp. \\
\hline 78 & Pochote & Ceiba pentandra \\
\hline 79 & Poleo & Mentha pulegium \\
\hline 80 & Quemadora, Ortiguilla & Tragia nepetaefolia ¿? \\
\hline 81 & Roble & Quercus sp. \\
\hline 82 & Romero, Romerillo & Rosmarinus officinalis \\
\hline 83 & Rosamarilla & Cochlospermum vitifolium \\
\hline 84 & Sacamanteca & Solanum bicolor \\
\hline 85 & Salvia & Salvia officinalis \\
\hline 86 & Sangregado & Jatropha curcas \\
\hline 87 & Sauce & Salix nigra \\
\hline 88 & Señorita & Asclepias curassavica \\
\hline 89 & Tachinole & Urera caracasana \\
\hline 90 & Taray & Tamarix gallica \\
\hline 91 & Tepehuaje & Lysiloma divaricada \\
\hline 92 & Toloache & Datura stramonium \\
\hline 93 & Tripa de zopilote & Cissus sicyoides \\
\hline 94 & Uvalamo & Vitex mollis \\
\hline 95 & Valeriana & Valeriana officinalis \\
\hline 96 & Vara blanca & Croton reflexolius \\
\hline 97 & Viejito & Cephalocereus sp. \\
\hline 98 & Vinorama & Acacia farnesiana \\
\hline 99 & Zapote dormilón & Casimiroa edulis \\
\hline 100 & Zarzaparrilla, Camote chino & Smilax aspera \\
\hline 101 & Zorrillo & Petiveria alliaceae \\
\hline
\end{tabular}

Además, estos resultados también aportan los nombres científicos, que como lo dicen Ocegueda et al. [11], son universales y no están limitados a una región en su lengua local, y con éllo se evita la confusión debida al hecho de que dos o más especies de plantas pueden tener el mismo nombre común.

\section{Conclusiones}

La expresión oral continua siendo la forma predominante de transmitir conocimientos, acerca de cuáles son las plantas medicinales silvestres o cultivadas, que a los pobladores 
de las comunidades rurales les ayudan a tratar y combatir muchas de las enfermedades que les aquejan durante su crecimiento y desarrollo.

El total de plantas silvestres medicinales mencionadas por los habitantes de las comunidades cuentan con una nomenclatura científica, lo que puede ser de utilidad para la transmisión de conocimientos científicos en las instituciones de educación de todos los niveles que se ofertan en Sinaloa, México y el mundo.

Los habitantes de las comunidades saben, de manera propia o a través de otras personas, de las propiedades de las plantas silvestres medicinales para curar diferentes enfermedades.

De manera general se observó que la forma de curar enfermedades con plantas medicinales en las comunidades situadas en las zonas de los altos del municipio de Culiacán, ubicadas en los sectores más pobres y desprotegidos de la población, forma parte de la cultura y tradiciones de las comunidades que se desarrollan sin mediar algún tipo de relación comercial, ya que la mayoría de las plantas medicinales son adquiridas de manera directa y natural en los montes, barrancos, arroyos y patios de las casas de sus lugares de origen.

La condición fresca de las plantas medicinales es la principal forma utilizada por los pobladores para aliviar algunas enfermedades, mismas especies que es necesario preservar para que las nuevas generaciones las conozcan y apliquen en el tratamiento de enfermedades que pueden curar.

Los conocimientos de herbolaria que tienen los habitantes de las comunidades en cuestión, son conocimientos empíricos que no van más allá de los adquiridos por la experiencia y sus prácticas cotidianas, ya que cuando se les solicitó explicación acerca del porqué las plantas medicinales ayudan para aliviar ciertas enfermedades, las respuestas fueron simples y superficiales.

\section{References}

[1] Gómez, A.R. (2012) Plantas medicinales en una aldea del Estado de Tabasco, México. Revista Fitotecnia Mexicana, 35, 43-49.

[2] Soto, R., Vega, G. and Tamajón, A.L. (2002) Instructivo técnico del cultivo de Cymbopogon citratus (D. C.) Stapf (caña santa). Revista Cubana de Plantas Medicinales, 7, 89-95.

[3] Leonti, M., Sticher, O. and Heinrich, M. (2003) Antiquity of Medicinal Plant Usage in Two Macro-Mayan Ethnic Groups (México). Journal of Ethnopharmacology, 88, 119-124. http://dx.doi.org/10.1016/S0378-8741(03)00188-0

[4] Canales, M., Hernández, T., Caballero, J., Romo de Vivar, A., Durán, A. and Lira, R. (2006) Análisis cuantitativo del conocimiento tradicional de las plantas medicinales en San Rafael, Coxcatlán, Valle de Tehuacán-Cuicatlán, Puebla, México. Acta Botanica Mexicana, 75, 21 43.

[5] Hernández, T., Canales, M., Caballero, J., Durán, A. and Liras, R. (2005) Análisis cuantitativo del conocimiento tradicional sobre plantas medicinales para el tratamiento de enfermedades gastrointestinales en Zapotitlán de las Salinas, Puebla, México. Interciencia, $30,17-27$.

[6] Ocampo, R. (1994) Estudio etnobotánico de las plantas empleadas por los indígenas en Talamanca, Costa Rica. Revista Forestal Centroamericana, 3, 16-21. 
[7] García, H.A.I., Morón, R.F.J. and Larrea, K.C. (2010) Plantas medicinales en revistas científicas de Cuba colonial y neocolonial. Revista Cubana de Plantas Medicinales, 15, 182 191.

[8] Morón, F., Sierra, P., Villán, J. and Martínez, M.J. (1991) Programa de medicina tradicional herbolaria en Cuba. Las plantas medicinales en la terapéutica. Revista Cubana de Medicina General Integral, 7, 276-284.

[9] M.S.P. (1999) Ministerio de Salud Pública. Programa Nacional de Medicina Tradicional y Natural. La Habana, 78-83.

[10] Germosén-Robineau, L. (2005) Farmacopea vegetal caribeña. 2da ed. Act. Editorial Universitaria UNAN, León, Nicaragua, 486 p.

[11] Ocegueda, S., Moreno, E. and Koleff, P. (2005) Plantas utilizadas en la medicina tradicional y su identificación científica. CONABIO. Biodiversitas, 62, 12-15.

[12] Linares, D., Bye, R. and Flores, F. (1999) Plantas medicinales de México. Usos, remedios y tradiciones. Instituto de Biología, UNAM, México, 155 p.

[13] Casas, A., Valiente-Banuet, A., Viveros, J.L., Dávila, P., Lira, R., Caballero, J., Cortés, L. and Rodríguez, I. (2001) Plant resources of the Tehuacán Valley, México. Economic Botany, 55, 129-166. http://dx.doi.org/10.1007/BF02864551

[14] Dávila, P., Arizmendi, M.C., Valiente-Banuet, A., Casas, A., Villaseñor, J.L. and Lira, S.R. (2002) Biological diversity in the Tehuacán-Cuicatlán Valley. Biodiversity and Conservation, 11, 421-442. http://dx.doi.org/10.1023/A:1014888822920

[15] Magaña, A.M.A., Gama, L. and Mariaca, R. (2010) El uso de las plantas medicinales en las comunidades Maya-Chontales de Nacajuca, Tabasco, México. Polibotánica, 29, 213-262.

[16] Fresquet, F.J.L. (2001) Uso popular de plantas medicinales en el medio urbano: la ciudad de Valencia. Medicina y Ciencias Sociales, 13, 1-15. http://www.uv.es/medciensoc

[17] Rojas, S. (2009) Medios de comunicación y políticas públicas: Producción de conocimiento en Colombia. Comunicación y Ciudadanía, 2, 104-113.

[18] Ramírez, C.E. (1998) Sobre los pasos de los ancestros. Arqueología Mexicana, 6, 64-71.

[19] García, L.F. (2005) La gran enciclopedia de las plantas curativas. Editorial Diana, México, D. F., 408 p.

[20] Ibarra, Ma.deJ., Cantú, P.C., Verde, M.J. and Oranday, A. (2009) Caracterización fitoquímica y efecto hipoglucemiante de Tecoma stans y su relación con la presencia del cromo como factor de tolerancia a la glucosa. Información Tecnológica, 20, 55-65. http://dx.doi.org/10.4067/S0718-07642009000500008

[21] Camacho, H.I.L., Cisneros, R.C., Uribe, B.M.J., Ríos, M.A. and Delgado, V.F. (2004) Antifungal Activity of Fruit Pulp Extract from Psidium sartorianum. Fitoterapia, 75, 401-404. http://dx.doi.org/10.1016/j.fitote.2004.01.004

[22] Pío-León, J.F., Díaz, C.S.P., López, L.M.A., Uribe, B.M.deJ., Willms, K., López, A.G., Montes, A.J. and Delgado, V.F. (2013) Actividad antibacteriana de extractos de frutos de nanchi (Byrsonima crassifolia L. Kunth), arrayán (Psidium sartorianum O. Berg Nied.) y áyale (Crescentia alata Kunth). Boletín Latinoamericano y del Caribe de Plantas Medicinales y Aromáticas, 12, 356-364.

[23] Montiel, H.M., Camacho, H.I.L., Ríos, M.A. and Delgado, V.F. (2004) Partial physicochemical and nutritional characterization of the fruit of Vitex mollis (Verbenaceae). Journal of Food Composition and Analysis, 17, 205-215. http://dx.doi.org/10.1016/j.jfca.2003.09.001

[24] Vega, A.R., Aguiar, H.H., Gutiérrez, G.J.A., Hernández, V.J.A., Vega, L.I.F. and Villaseñor, 
J.L. (2000) Endemismo regional presente en la flora del municipio de Culiacán, Sinaloa, México. Acta Botanica Mexicana, 53, 1-15.

[25] Flores, C.L.M., Arzola, G.J.F., Ramírez, S.M. and Osorio, P.A. (2012) Repercusiones del cambio climático global en el estado de Sinaloa, México. Revista Colombiana de Geografía, 21, 115-129.

Submit or recommend next manuscript to OALib Journal and we will provide best service for you:

- Publication frequency: Monthly

- 9 subject areas of science, technology and medicine

- Fair and rigorous peer-review system

- Fast publication process

- Article promotion in various social networking sites (LinkedIn, Facebook, Twitter, etc.)

- Maximum dissemination of your research work

Submit Your Paper Online: Click Here to Submit

Or Contact service@oalib.com 